IJMMS 31:10 (2002) 635-637

PII. S0161171202013078

http://ijmms.hindawi.com

(c) Hindawi Publishing Corp.

\title{
POWERS OF COMMUTATORS AS PRODUCTS OF SQUARES
}

\section{AKHAVAN-MALAYERI}

Received 2 May 2001 and in revised form 13 January 2002

Let $F$ be a free group and $x, y$ be two distinct elements of a free generating set, then $[x, y]^{n}$ is not a product of two squares in $F$, and it is the product of three squares. We give a short combinatorial proof.

2000 Mathematics Subject Classification: 20F12, 20 F99.

1. Introduction. It has been shown by Lyndon and Newman [2] that in the free group $F=F(x, y)$, freely generated by $x, y$, the commutator $[x, y]$ is never the product of two squares in $F$, although it is always the product of three squares. Let $\gamma \in F^{\prime}$, the minimal number of squares which is required to write $\gamma$ as a product of squares in $F$ is called the square length of $\gamma$ and denoted by $\operatorname{Sq}(\gamma)$. Here we consider more general case, that is, $\operatorname{Sq}[x, y]^{n}, n \in \mathbb{N}$.

Throughout this paper, $x^{y}$ means $y x y^{-1} ;[x, y]=x y x^{-1} y^{-1} ; G^{\prime}$ denotes the derived subgroup of $G$, and $\gamma_{m}(G)$ denotes the $m$ th term of the lower central series of $G$.

2. Main result. The main result of this note is the following theorem.

THEOREM 2.1. Let $F$ be a free group and let $x, y$ be two distinct elements of a free generating set, then $\mathrm{Sq}[x, y]^{n}=3$ if $n \in \mathbb{N}$ is odd, and $\mathrm{Sq}[x, y]^{n}=1$ if $n$ is even.

Proof. In the case when $n$ is even, the result is clear. Let $n$ be an odd integer. First, we show that $[x, y]^{n}$ can be written as a product of 3 squares in $F$. Put $[x, y]=W$, then we can check the following identity:

$$
W^{2 k+1}=[x, y]^{2 k+1}=\left(\left(W^{k} x y\right)^{W^{k}}\right)^{2}\left(W^{k} y^{-1}\right)^{2}\left(\left(W^{-k} x^{-1}\right)^{y}\right)^{2} .
$$

In the case $k=0$, we get

$$
[x, y]=(x y)^{2}\left(y^{-1}\right)^{2}\left(\left(x^{-1}\right)^{y}\right)^{2},
$$

hence

$$
\mathrm{Sq}[x, y]^{n} \leq 3,
$$

hence to complete the proof it is enough to show that

$$
\mathrm{Sq}[x, y]^{n} \neq 2 .
$$


The case $n=1$ was proved by Lyndon and Newman [2], so we prove that $W^{2 k+1} \neq a^{2} b^{2}$ for any $k \in \mathbb{N}$ and $a, b \in F$. Lyndon and Schützenberger [3] proved that

$$
a^{M}=b^{N} c^{P}, \quad M, N, P \geq 2,
$$

implies that $a, b$, and $w$ all lie in a cyclic subgroup. Therefore, all components $a, b$, and $w$ of a solution of the equation $W^{r}=a^{2} b^{2}$, for $r \geq 2$, must belong to the cyclic subgroup generated by $W$. Hence, we reduce the problem to the case of rank two, we may assume $F=F(x, y)$ to be the free group of rank two freely generated by $x, y$, and suppose $a^{2} b^{2}=W^{r}$ for some $r \in \mathbb{Z}$, then

$$
a^{2} b^{2} \equiv(a b)^{2} \bmod F^{\prime}
$$

Since $a^{2} b^{2} \in F^{\prime},(a b)^{2} \in F^{\prime}$, hence $a b \in F^{\prime}$ and $a=u b^{-1}$ for some $u \in F^{\prime}$. Now $a^{2}=$ $\left(u b^{-1}\right)^{2}=u u^{b^{-1}} b^{-2}$, hence $u u^{b^{-1}}=W^{r}$ and $W^{r} \equiv u^{2}\left(\bmod \gamma_{3}(F)\right)$.

But $\gamma_{2}(F) / \gamma_{3}(F) \cong C_{\infty}$ and it is generated by $W=[x, y]$. Since $W$ is the generator of $\gamma_{2}(F) \bmod \gamma_{3}(F), u^{2} \equiv W^{r}$ has solution if and only if $r$ is even, hence we proved that $W^{2 k+1} \neq a^{2} b^{2}$ for any $k \in \mathbb{N}$.

We have the following notations.

(1) In a similar way $a^{n} b^{n}=W^{r}$ for some $r \in \mathbb{Z}$ implies that

$$
\begin{gathered}
a^{n}=\left(u b^{-1}\right)^{n}=u u^{b^{-1}} u^{b^{-2}} \cdots u^{b^{-(n-1)}} b^{-n}, \\
a^{n} b^{n}=u u^{b^{-1}} u^{b^{-2}} \cdots u^{b^{-(n-1)}},
\end{gathered}
$$

for some $u \in F^{\prime}$. And we have

$$
u^{n} \equiv W^{r} \bmod \gamma_{3}(F)
$$

so, $n \mid r$, hence, if $n$ is not a multiple of $r$, then $a^{n} b^{n} \neq W^{r}$.

(2) In $F(x, y), \mathrm{Sq}[x, y]^{n}=3$ for any odd number $n \in \mathbb{N}$. But there exists commutators with square length equals to two. Obviously, $\left[h^{2}, g\right]$ and $\left[h, g^{2}\right]$ are products of two squares, and a nontrivial commutator is never a square [4]. Thus $\mathrm{Sq}\left[h^{2}, g\right]=$ $\mathrm{Sq}\left[h, g^{2}\right]=2$.

But it is not the only case in which the square length of a commutator is two, as shown by Comerford and Edmundss in [1].

ACKNOWLEDGMENTS. This research was in part supported by a grant from the Institute for Studies in Theoretical Physics and Mathematics (IPM). The author is indebted to Professor A. Rhemtulla of the University of Alberta for various helpful communications concerning the matters discussed in this note, and the author would like to thank the referees who have patiently read and verified this note, and also suggested valuable comments. The author also likes to acknowledge the support of the IPM. 


\section{REFERENCES}

[1] L. P. Comerford Jr. and C. C. Edmunds, Products of commutators and products of squares in a free group, Internat. J. Algebra Comput. 4 (1994), no. 3, 469-480.

[2] R. C. Lyndon and M. Newman, Commutators as products of squares, Proc. Amer. Math. Soc. 39 (1973), 267-272.

[3] R. C. Lyndon and M. P. Schützenberger, The equation $a^{M}=b^{N} c^{P}$ in a free group, Michigan Math. J. 9 (1962), 289-298.

[4] M. P. Schützenberger, Sur l'équation $a^{2+n}=b^{2+m} c^{2+p}$ dans un groupe libre, C. R. Acad. Sci. Paris 248 (1959), 2435-2436 (French).

M. AKHAVAN-Malayeri: AZZAhra UniVersity, VANaK, TEHran 19834, Iran

E-mail address: mma7ayer@azzahra.ac.ir 


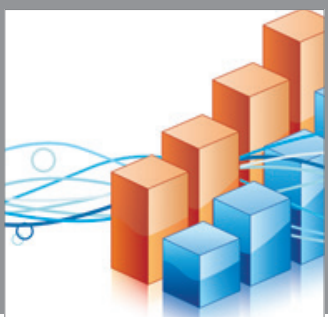

Advances in

Operations Research

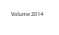

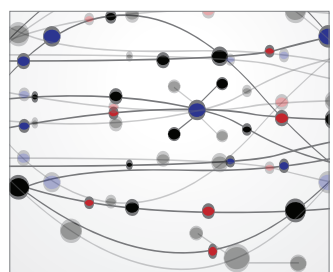

\section{The Scientific} World Journal
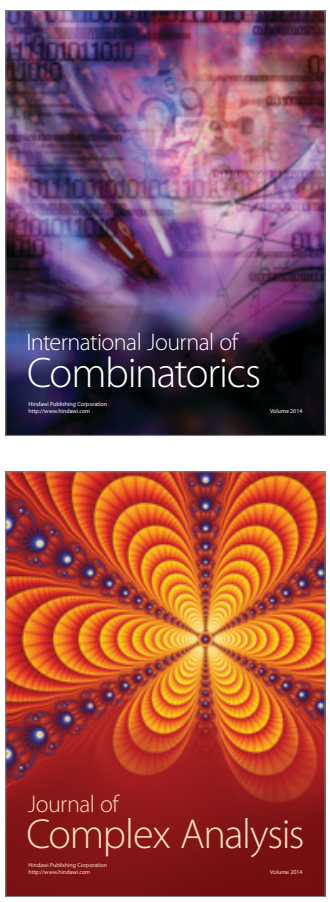

International Journal of

Mathematics and

Mathematical

Sciences
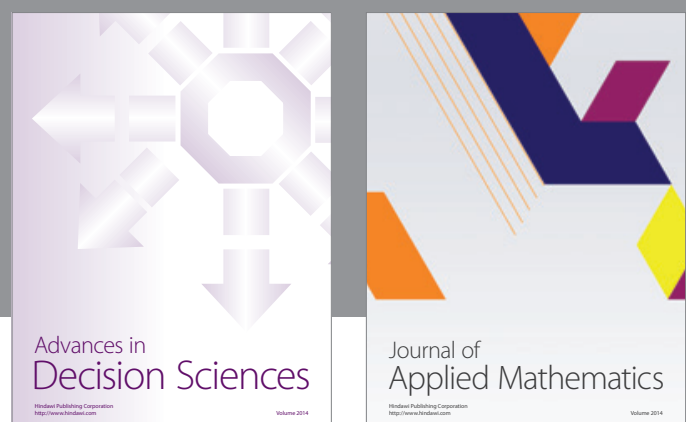

Journal of

Applied Mathematics
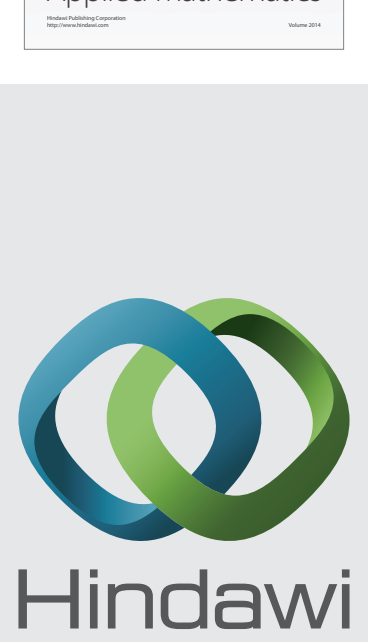

Submit your manuscripts at http://www.hindawi.com
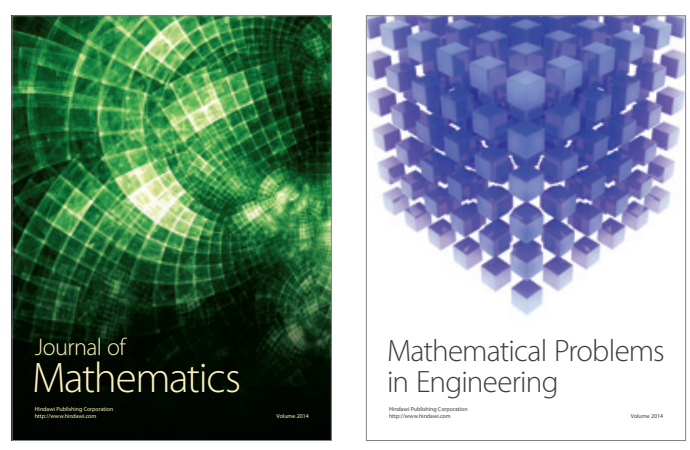

Mathematical Problems in Engineering
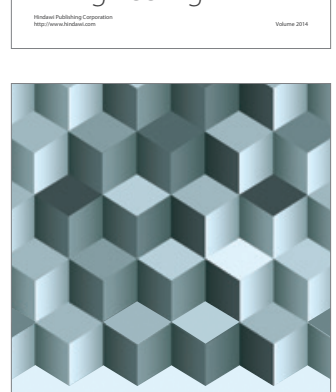

Journal of

Function Spaces
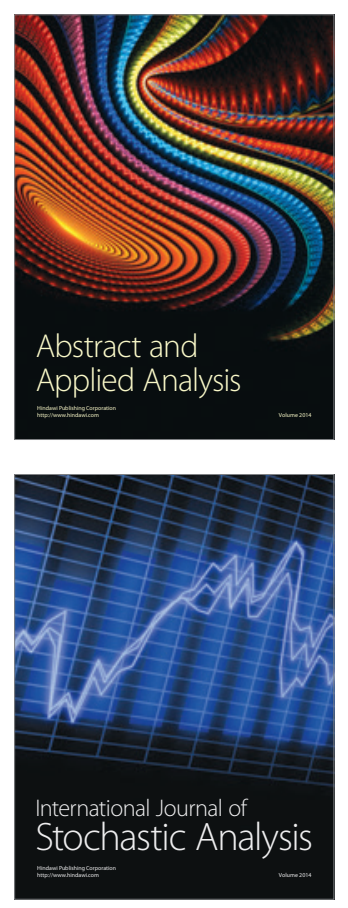

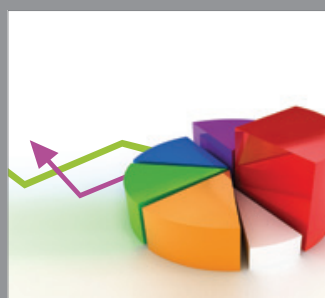

ournal of

Probability and Statistics

Promensencen
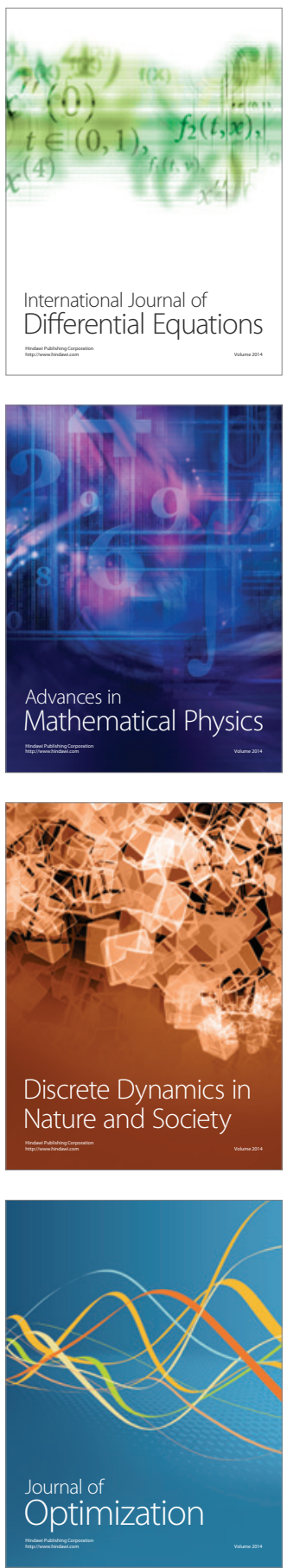